\title{
Eau De Consommation Et Maladies Hydriques Dans La Commune De Lokossa Au Sud-Ouest De La Republique Du Benin (Afrique De L'ouest)
}

\author{
Ahouansè Sènan Dimitri Miriac, \\ Laboratoire Pierre Pagney : Climat, Eau, Ecosystèmes et Développement \\ (LACEEDE), République du Bénin \\ Agossou Noukpo, Professeur Titulaire \\ Laboratoire d'Aménagement Régional \& Développement (LARD), \\ République du Bénin \\ Houssou Sègbè Christophe, Professeur Titulaire \\ Laboratoire Pierre Pagney : Climat, Eau, Ecosystèmes et Développement \\ (LACEEDE), République du Bénin
}

Doi:10.19044/esj.2020.v16n15p393 URL:http://dx.doi.org/10.19044/esj.2020.v16n15p393

\section{Résumé}

Les populations de la commune de Lokossa sont confrontées au problème d'approvisionnement en eau potable. La présente étude porte sur l'influence de l'eau de consommation sur la santé des populations de la commune de Lokossa. Pour réussir cette étude, les sources d'eau ont été répertoriées et des échantillons d'eau ont été analysés du point de vue physicochimique et bactériologique au laboratoire de la Direction Générale de l'Eau. La recherche documentaire et les investigations socio-anthropologiques ont permis d'avoir les données exploitées dans cette étude. Les analyses faites montrent que l'eau consommée par les populations est souvent polluée. Les mauvaises pratiques humaines contribuent à la dégradation de la qualité de l'eau et au développement des larves de moustiques. Cette situation est à l'origine des maladies hydriques (diarrhées et paludisme) qui sévissent dans le milieu. Il urge donc de mener des politiques et stratégies concertées en vue de sensibiliser les populations sur les dangers de leurs mauvaises pratiques sur la santé.

Mots Cles : Eau De Consommation, Maladies Hydriques, Lokossa, Sud-Ouest De La République Du Bénin, Afrique De l'Ouest 


\title{
Water Consumption and Waterborne Diseases in the Commune of Lokossa in Southwest of the Republic of Benin (West Africa)
}

\author{
Ahouanse Senan Dimitri Miriac, \\ Laboratoire Pierre Pagney : Climat, Eau, Ecosystèmes et Développement \\ (LACEEDE), République du Bénin \\ Agossou Noukpo, Professeur Titulaire \\ Laboratoire d'Aménagement Régional \& Développement (LARD), \\ République du Bénin \\ Houssou Segbe Christophe, Professeur Titulaire \\ Laboratoire Pierre Pagney : Climat, Eau, Ecosystèmes et Développement \\ (LACEEDE), République du Bénin
}

\begin{abstract}
The populations of the commune of Lokossa are confronted to drinking water supply issues. This study focuses on how water consumption affects the health of the populations in the commune of Lokossa. To make this study a success, water sources were identified and a physicochemical and bacteriological analysis was conducted on the samples of water in the laboratory of the General Directorate for Water. The documentary research and socio-anthropological investigations provided the data used in this study. The analysis revealed that the water consumed by the populations is often polluted. Poor human practices contribute to the degradation of the water quality and the development of mosquito larvae. This situation is at the root of the waterborne diseases (diarrhoea and malaria) that prevail in the region. Therefore, concerted policies and strategies must be pursued in order to sensitize the populations on the impacts of their bad practices on health.
\end{abstract}

Keywords : Water Consumption, Waterborne Diseases, Lokossa, Southwest Of The Republic Of Benin, West Africa

\section{Introduction}

L'eau est une ressource indispensable à la vie, aussi bien végétale, animale, qu'humaine. L'accès à une eau de consommation saine est incontestablement bénéfique pour la santé. Dans le monde, 1,1 milliard de personnes n'ont pas un accès suffisant à l'eau potable (CAE, 2010). 
Au Bénin les populations ont des difficultés d'accès à l'eau de consommation. Lorsqu'elle est accessible, l'eau est souvent sujette à une contamination chimique et/ou bactériologique (Sohounou, 2018). La contamination fécale de l'eau de consommation est d'origine humaine ou animale, directe ou indirecte (Lalèyè, 2016). L'eau de mauvaise qualité, utilisée dans les ménages est à l'origine des maladies hydriques constatées chez les membres de ces ménages (Babadjidé, 2008).

La Commune de Lokossa n'est pas en marge de cette situation. Le présent travail s'intéresse à l'influence de l'eau de consommation sur la santé des populations dans la commune de Lokossa en République du Bénin.

\section{1- $\quad$ Presentation Du Secteur D'etude}

La commune de Lokossa située au nord-ouest du département du Mono, est l'une des six (06) subdivisions administratives que compte ce département. Elle est comprise entre $6^{\circ} 36^{\prime}$ et $6^{\circ} 45^{\prime}$ de latitude nord et entre $1^{\circ} 35^{\prime}$ et $1^{\circ} 50^{\prime}$ de longitude est. Elle couvre une superficie de $260 \mathrm{~km}^{2}$, ce qui représente $16 \%$ de la superficie du département du Mono $\left(1605 \mathrm{~km}^{2}\right)$ et $0,23 \%$ de la superficie totale de la République du Bénin qui est de $114.763 \mathrm{~km}^{2}$ (RGPH, 2013). Limitée au Nord par la commune de Dogbo dans le Couffo, au Sud par les communes d'Athiémé et de Houéyogbé, à l'Est par celle de Bopa et à l'Ouest par le territoire togolais, cette commune a son chef-lieu distant de $106 \mathrm{~km}$ de Cotonou, capitale économique du Bénin. Sur le plan administratif, la commune est divisée en cinq (05) arrondissements que sont: Lokossa, Agamè, Koudo, Houin et Ouèdème-Adja.

La figure 1 présente la situation géographique et administrative de la commune Lokossa. 


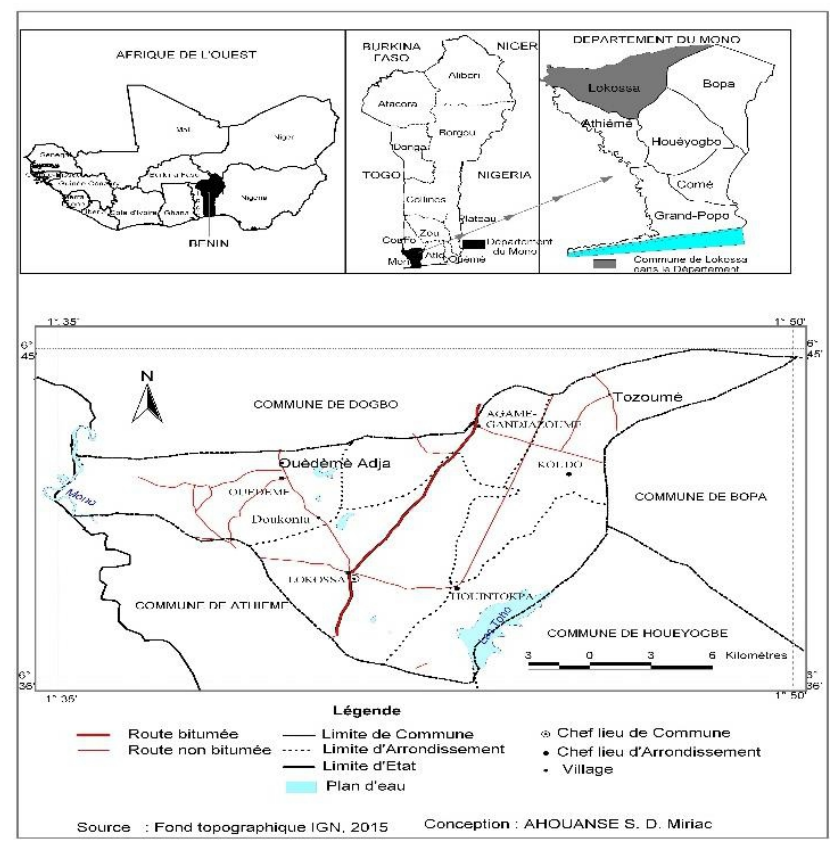

Figure 1 : Situation géographique et administrative de la commune de Lokossa (République du Bénin)

\section{2- Donnees, Techniques Et Methodes}

Dans le cadre de cette étude, une série de données a été collectée grâce à plusieurs techniques. Différentes méthodes ont permis le traitement des données collectées.

\section{2-1 Données}

Il s'agit des données démographiques, des données socioanthropologiques, des paramètres physico-chimiques et microbiologiques et des données épidémiologiques.

- Les données démographiques concernent les données issues du Recensement Général de la Population et de l'Habitation (RGPH) 2013. Elles ont été utiles dans la détermination la taille de l'échantillon des ménages à enquêter.

- Les données socio-anthropologiques pour une appréciation endogène de la qualité de l'eau de consommation et les risques sanitaires.

- Les données sur les paramètres physico-chimiques et microbiologiques (température, $\mathrm{pH}$, conductivité, couleur, ammonium, nitrates, nitrites, sulfates, fluores, iodure, fer total, chlorures, bicarbonates, magnésium, calcium, dureté totale, alcalinité, coliformes totaux, coliformes fécaux, streptocoques fécaux) pour apprécier la qualité des eaux consommées. 
- Les données épidémiologiques sur une période de 2002-2018 dans la Commune de Lokossa, obtenues à la Direction Départementale de la Santé Mono, au Ministère de la Santé (MS) à partir du SNIGS (Système National Intégré de Gestion Sanitaire) et complétées par les enquêtes de terrain de 2015 à 2018.

\section{2-2 Techniques de collecte des données}

Sur le terrain, l'observation directe, l'entretien semi-direct avec les personnes ressources, l'entretien direct avec les ménages et les focus groups ont permis d'appréhender le niveau de couverture des localités en infrastructures d'approvisionnement en eau potable et les difficultés d'accès à l'eau de consommation. Les outils utilisés sont le questionnaire-ménage, le guide d'entretien pour les personnes ressources et la grille d'observation. En ce qui concerne l'échantillonnage :

- Pour déterminer la taille de l'échantillon des ménages à enquêter, la méthode probabiliste de Marien et Beaud (2003), a été appliquée à l'effectif de la population de 2013. Ainsi de 20822 ménages (INSAE, 2016) a été tiré un échantillon de 393 ménages suivant le protocole statistique 1 de Marien et Beaud (2003) :

$$
\mathbf{n}=\frac{\mathrm{N}(\text { Effectif total }) \times 400}{\mathrm{~N}(\text { Effectif total })+400} \times 100
$$

avec $\mathrm{n}=$ taille de l'échantillon et $\mathrm{N}=$ effectif des ménages de la commune de Lokossa selon le RGPH de 2013.

$\mathrm{n}=(20822 \times 400) /(20822+400)$

$\mathrm{n}=8328800 / 21222$

$\mathrm{n}=392,46 ; \mathbf{n}=393$ ménages.

La répartition de la taille de l'échantillon au niveau de chaque arrondissement est présentée dans le tableau I.

Dans le souci de respecter une proportionnalité des différentes classes de l'échantillon constitué par arrondissement, un coefficient, $\mathbf{K}=\mathbf{n} / \mathbf{N}$ est appliqué. Avec $: \mathrm{n}=$ taille de l'échantillon et $\mathrm{N}=$ ménage total de la commune. Ainsi, $K=393$ / $20822 ; K=0,01887 ; K=0,019$

Le nombre représentatif de chaque catégorie est obtenu en multipliant le nombre de ménages de chaque arrondissement par ce coefficient $\mathrm{K}$.

Les effectifs de l'échantillon de chaque arrondissement sont présentés dans le tableau I suivant : 
Tableau I : Echantillonnage des ménages enquêtés par arrondissement

\begin{tabular}{ccc}
\hline Arrondissements & Ménages en 2013 & Ménages enquêtés \\
\hline Agamè & 3489 & 66 \\
Houin & 1642 & 31 \\
Koudo & 2888 & 55 \\
Lokossa & 10034 & 189 \\
Ouèdèmè & 2769 & 52 \\
\hline Total & 20822 & 393 \\
\hline
\end{tabular}

Source : Marien et Beaud, 2003 ; INSAE, 2016.

Au total, trois cent quatre-vingt-treize (393) individus, à raison d'une personne par ménage, sont interrogés.

- Pour les entretiens semi-structurés, trente-six (36) personnes ressources choisies de façon aléatoire ont été interrogées.

- Quant aux focus groups, ils ont permis d'obtenir des clarifications sur les informations recueillies individuellement (données épidémiologiques). Au total, dix (10) focus groups ont été organisés et quatre-vingt-onze (91) personnes y ont participé.

- Un échantillon de 25 prélèvements d'eau a été donc retenu. Les sites de prélèvement ont été choisis en tenant compte des paramètres morpho-structuraux, de l'accessibilité et de la pression humaine exercée sur les points d'eau. A la source, les échantillons ont été prélevés à la sortie du réseau. Pendant le transport, ils ont été faits dans tous les types de récipients rencontrés lors de l'enquête. Les échantillons faits au stockage de l'eau à domicile sont prélevés dans les jarres ou les plastiques qui servent de retenue d'eau pour les ménages.

\section{2-3 Méthodes de traitement des données}

2-3-1 Méthodes d'analyse des paramètres physico-chimiques et bactériologiques des échantillons d'eau

Les échantillons ont été analysés au laboratoire de la Direction Générale de l'Eau à Cotonou. Toutes les analyses ont été effectuées selon les méthodes standardisées de l'American Public Health Association (APHAAWWA-WPCF, 1994). En effet, les analyses physico-chimiques ont été effectuées suivant les méthodes de dosage telles que la volumétrie et la spectrophotométrie. Les paramètres sont dosés par les méthodes potentiométriques $(\mathrm{pH})$, électro métrique (couleur, conductivité/TDS). La volumétrie (méthode de dosage) a été appliquée à l'analyse quantitative du calcium, du magnésium, des chlorures et des bicarbonates. En effet, les teneurs en calcium, magnésium des échantillons sont déterminées par complexométrie, par titrage avec l'acide Ethylène-Diamine Tétra Acétique (EDTA). La teneur en chlorures des échantillons est déterminée par 
argentométrie (principe de Mohr par titrage avec le nitrate d'argent). Enfin la teneur en bicarbonates des échantillons est déterminée par dosage acidobasique, par titrage avec l'acide sulfurique. La spectrophotométrie a été utilisée pour l'analyse quantitative des nitrates, nitrites, ammonium, fluorures, fer total et sulfates par dosage colorimétrique.

Les analyses bactériologiques concernent les coliformes totaux, les coliformes fécaux et les streptocoques fécaux. Les méthodes de recherche et de dénombrement des germes utilisées sont celles de référence retenue par la norme. Il s'agit de la préparation du local, de la préparation des milieux de culture et du matériel de travail, de la reconstitution de la poudre du Rapid E. coli (REC), de la reconstitution de la gélose de Slanetz-Bartley pour les streptocoques fécaux, de la reconstitution du Baird-Parker, de l'ensemencement, de l'incubation et de la lecture.

\section{- Préparation du local}

Le laboratoire est nettoyé et la paillasse correctement désinfectée avec une solution désinfectante (solution à base de chlore ou d'alcool à $70 \%$ ).

- Préparation des milieux de culture et du matériel de travail

Le milieu de culture utilisé pour la recherche des Coliformes (totaux fécaux et E. Coli) est le rapid E. coli, le Baird Parker pour les clostridium aureus et celui des Streptocoques fécaux est le milieu Slanetz-Bartley. La poudre REC, de Baird Parker et celle de Slanetz sont reconstituées en suivant les indications du fabriquant.

- Reconstitution de la poudre du Rapid E. coli (REC)

- Ajouter $37 \mathrm{~g}$ de poudre à $1 \mathrm{~L}$ d'eau distillée ;

- Porter doucement à ébullition jusqu à dissolution complète ;

- Stériliser pendant 15 minutes à $121^{\circ} \mathrm{C}$ à $1^{\prime}$ autoclave ;

- Laisser refroidir à environ $50^{\circ} \mathrm{C}$;

- Bien mélanger et répartir dans les boîtes de pétri stériles.

- Reconstitution de la gélose de Slanetz-Bartley pour les streptocoques fécaux

- $\quad$ Ajouter $41.5 \mathrm{~g}$ de poudre à $1 \mathrm{~L}$ d'eau distillée ;

- Porter doucement à ébullition jusqu à dissolution complète ;

- Stériliser pendant 15 minutes à $121^{\circ} \mathrm{C}$ à $1^{\prime}$ autoclave ;

- $\quad$ Laisser refroidir à environ $50^{\circ} \mathrm{C}$.

- Reconstitution du Baird-Parker

Mettre en suspension $58 \mathrm{~g}$ de poudre dans $950 \mathrm{ml}$ d'eau distillée ou déminéralisée. Porter à ébullition lentement en agitant jusqu'à dissolution complète. Répartir en flacon, à raison de :

- $95 \mathrm{ml}$ par flacon pour Baird-Parker avec le jaune d'œuf ;

- Stériliser à l'autoclave à $212^{\circ} \mathrm{C}$ pendant $15 \mathrm{mn}$.

- Laisser refroidir à environ $50^{\circ} \mathrm{C}$;

Pendant que le milieu se refroidit, il faut : 
- Apprêter les boîtes de pétrie (pour les échantillons à ensemencer par incorporation) en inscrivant les numéros, la date, le milieu, la température d'incubation;

- Apprêter les boîtes de pétrie pour couler le milieu sur lequel sera déposée la membrane cellulosique ;

- Stériliser à l'aide du feu d'un bec bunsen ou d'un pistolet à gaz, la rampe de filtration (en acier inoxydable) ;

- Apprêter les pinces, la membrane cellulosique de $0,45 \mu \mathrm{m}$ et autres éléments.

Dès que tout est pratiquement prêt, il faut commencer les incorporations et les filtrations.

- Méthode d'ensemencement

Les eaux ont été incubées par la méthode de filtration de $100 \mathrm{ml}$ d'échantillon et par incorporation.

- Incubation

Les échantillons sont incubés à $37^{\circ} \mathrm{C}$ pour les Coliformes totaux, les Clostridium aureus et les Streptocoques fécaux et à $44^{\circ} \mathrm{C}$ pour les Coliformes fécaux et les Escherichia coli dans des incubateurs préréglés aux températures indiquées plus haut. La boîte de pétrie est retournée (couvercle en bas) avant l'incubation pour éviter que les vapeurs d'eau ne dispersent les colonies formées empêchant ainsi de faire une lecture correcte.

- Lecture

Après 24 heures d'incubation, les Colonies de Coliformes qui sont bleues sont les Coliformes totaux ou fécaux selon que cela soit sorti de l'incubateur de $37^{\circ} \mathrm{C}$ ou $44^{\circ} \mathrm{C}$. Les colonies d'Escherichia coli sont violettes. Les Streptocoques fécaux apparaissent rouges sur le milieu Slanetz-Bartley après 24 à 48 heures et les Clostridium eux sont noir avec halo. Une confirmation sur le milieu Bile Esculine est indispensable pour les Streptocoques fécaux. Les résultats obtenus ont été traités, analysés et comparés aux normes de l'ABéNor (2003) et de l'OMS (2002).

\section{2-3-2 Analyse de l'état de santé des populations}

Les statistiques de cas de maladies enregistrés dans la Zone Sanitaire Lokossa-Athiémé de 2002-2018 et particulièrement les relevés épidémiologiques des aires sanitaires de Lokossa ont été traitées pour mettre en évidence les maladies hydriques dominantes et les confronter avec les risques sanitaires liés à la consommation d'eau polluée. L'état de santé des populations est apprécié à travers l'analyse des données sanitaires (sur les maladies diarrhéiques et le paludisme) collectées dans les centres de santé de la commune et dans le service en charge des statistiques du Ministère de la Santé. Des tableaux et figures d'illustrations sont produits à partir du regroupement des données sur les maladies. 


\section{2-3-3 Méthode d'analyse des statistiques épidémiologiques}

Les statistiques épidémiologiques ont été traitées suivant la prévalence. La prévalence est un indicateur statique de morbidité. Elle se définit comme la proportion d'un nombre de cas d'une maladie à un instant donné sur la population dont sont issus ces cas (Ancelle, 2011). Elle est déterminée par la formule 2 .

Prévalence $=\frac{\text { Nombre de cas }(\text { ancien }+ \text { nouveau }) \mathrm{d}^{\prime} \text { une maladie à un instant } \mathrm{t}}{\text { Population cible à un instant } \mathrm{t}} \times \mathbf{1 0 0 0}$

Ce paramètre renseigne sur l'importance d'une maladie ou d'une infection dans une population à un moment donné.

\section{3- $\quad$ Resultats Et Discussions}

3-1 Appréciations endogènes de la qualité de l'eau de consommation et méthodes endogènes de traitement de l'eau

Les investigations menées à travers les interviews, ont permis d'évaluer le niveau de connaissance des populations de la qualité des eaux consommées. Au nombre des moyens plus pertinents pour apprécier la qualité de l'eau de consommation, se trouvent en bonne place les paramètres organoleptiques (odeur, saveur, couleur) et des indicateurs visibles (particules fines, débris et microbes).

\section{3-1-1 Concept local d'eau propre : un critère d'appréciation de la $p$ otabilité de l'eau}

Généralement, une eau limpide sans charge de boue, dépourvue de débris, de particules fines et des larves de tout genre est vue comme potable par les populations. La présence de l'un de ces éléments dans l'eau lui attribue le qualificatif d'eau sale mais ne signifie guère, à l'exception du cas des larves, qu'elle est systématiquement polluée. Lorsqu'au repos, elle peut être débarrassée de ces impuretés, l'eau est de nouveau considérée propre et jugée potable. Les larves dans l'eau sont craintes car supposées qu'une fois consommées, elles se développeront dans les intestins pour entraîner les maux de ventre ou les ascaris. La figure 25 présente les proportions de populations par rapport à la qualification de l'eau à travers son aspect physique.

La figure 2 indique que selon $83 \%$ de la population cible, l'eau est potable lorsqu'elle est propre. Par contre une frange de $14 \%$ de cette même population affirme que l'eau est sale quand elle est polluée tandis que minoritairement $3 \%$ estime que l'eau consommée quand bien même propre et non traitée, est polluée. Cette considération du critère lié à l'eau potable est l'un des fondements qui accrochent les populations à l'utilisation de l'eau de toute nature. 


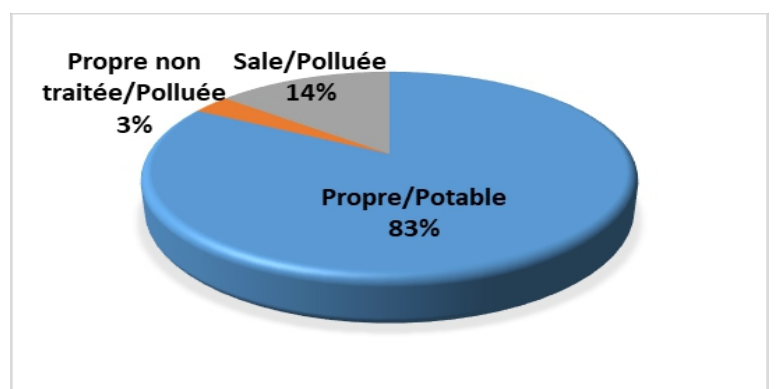

Figure 22: Appréciation de la qualité de l'eau par la propreté

Source : Résultats des travaux d'enquête de terrain, (Ahouansè, 2015, 2016, 2017, 2018) et traitements statistiques

\section{3-1-2 Odeur de l'eau}

L'odeur de l'eau n'est pas le critère dominant de qualification des eaux de consommation dans le milieu d'étude. La figure 3 illustre la réaction des enquêtés par rapport à la potabilité de l'eau en fonction de son odeur.

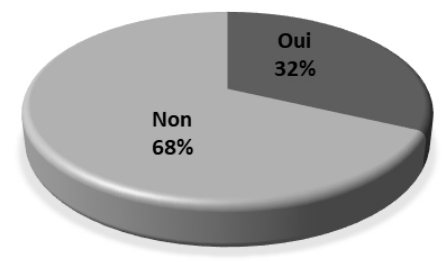

Figure 3 : Appréciation de la qualité de l'eau par son odeur

Source : Résultats des travaux d'enquête de terrain, (Ahouansè, 2015, 2016, 2017, 2018) et traitements statistiques

Une proportion de $68 \%$ des ménages interrogés considèrent que l'odeur n'est pas un indice de la mauvaise qualité de l'eau. Pour elles, l'odeur d'une eau peut découler de la nature de la couche de roche qui abrite la nappe phréatique ou des racines des arbres et herbes. Pour renchérir leur déclaration, certaines personnes précisent que les substrats issus de la roche, des racines d'arbres et des herbes sont enrichissants et ont des vertus thérapeutiques, augmentant ainsi les éléments nutritifs de l'eau. Au contraire, environ le tiers des personnes interrogées, soit $32 \%$ de l'ensemble, sous-tendent la qualité de l'eau à son odeur. Dans ce cadre, est considérée comme non potable toute eau dont l'odeur est très répugnante. Ces $32 \%$ de personnes enquêtées fondent leurs arguments sur le fait qu'une mauvaise odeur résulte de la décomposition des animaux et végétaux noyés, des déchets humains et des ordures évacuées vers l'eau qui, du coup, dégradent la qualité des eaux et peuvent parfois entraîner un empoisonnement. Il y ressort donc que l'odeur de l'eau ne constitue pas un élément prépondérant dans l'appréciation de la qualité de l'eau. 


\section{3-1-3 Saveur de l'eau}

La saveur des eaux a été nuancée par rapport à sa source dans l'appréciation de la qualité de l'eau. Selon les sujets enquêtés, la saveur peut être le signe de pollution de l'eau pour le cas des marigots et de non pollution pour les eaux profondes. Dans l'ensemble, la saveur n'est pas déterminante dans la qualification des eaux en milieu rural tel que le montre la figure 4.

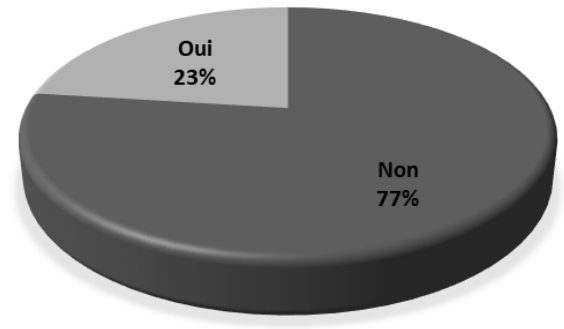

Figure 4 : Appréciation de la qualité de l'eau par la saveur

Source : Résultats des travaux d'enquête de terrain, (Ahouansè, 2015, 2016, 2017, 2018) et traitements statistiques

Selon $77 \%$ de la population enquêtée (figure 4), la qualité de l'eau n'est pas imputable à la saveur. Pour cette catégorie de population, l'eau peut prendre la saveur des racines des arbres qui immergent son lit ou des constituants des roches traversées par la nappe phréatique. Par contre, $23 \%$ considèrent que la saveur est un indicateur de mauvaise qualité de l'eau pour la consommation.

\section{3-1-4 Couleur de l'eau}

La couleur de l'eau est un paramètre organoleptique controversé de catégorisation de l'eau. La figure 5 met en exergue l'appréciation de la qualité de l'eau par la couleur issue de l'avis des ménages interrogés. Suivant cette appréciation, $48 \%$ des populations enquêtées pensent que la couleur peut permettre de dire qu'une eau est potable ou non. Une eau potable, selon elles, doit être limpide autrement dit sans couleur.

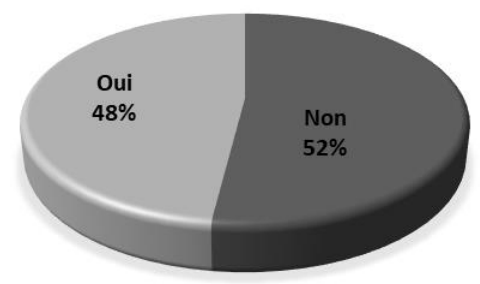

Figure 5 : Appréciation de la qualité de l'eau par la couleur

Source : Résultats des travaux d'enquête de terrain, (Ahouansè, 2015, 2016, 2017, 2018) et traitements statistiques 
Cette assertion n'est pas partagée par $52 \%$ des enquêtés qui ne lient pas systématiquement la qualité de l'eau à sa couleur. Il ressort, néanmoins, de leur justification que ceci est seulement valable pour les eaux des sources profondes qui épousent la couleur de la roche mère.

\section{3-1-5 Présence des débris}

La présence des débris dans l'eau est bien considérée pour sa classification. La figure 6 illustre l'avis des populations interviewées sur une eau contenant des débris.

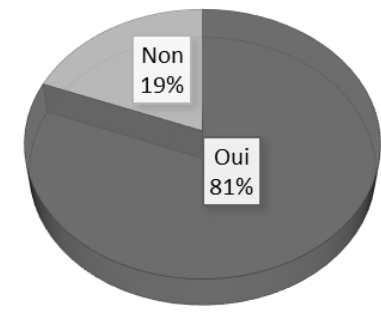

Figure 6: Appréciation de la qualité de l'eau par la contenance de débris

Source : Résultats des travaux d'enquête de terrain, (Ahouansè, 2015, 2016, 2017, 2018) et traitements statistiques

L'examen de la figure 6 révèle que $81 \%$ des populations interrogées pensent que l'eau contenant des débris des animaux et végétaux est à priori une eau malpropre, donc de mauvaise qualité. La présence de débris dans une eau est considérée comme la source première de classement de l'eau de consommation dans le secteur d'étude.

Au demeurant, la population de la Commune de Lokossa assimile une eau de bonne qualité à sa propreté. L'eau polluée est celle qui est sale et contenant des débris végétaux, animaux ou des ordures ménagères.

\section{3-1-6 Méthodes endogènes de traitement de l'eau de consommation}

L'objectif fondamental du traitement de l'eau est de protéger les consommateurs des micro-organismes pathogènes et de l'impureté désagréable ou dangereuse pour la santé. L'inefficacité des méthodes amène les populations à consommer une eau qui affecte leur état de santé (Blalogoe, 2002). Les différents procédés ne détruisent pas toutes les bactéries de l'eau de consommation (Hedible, 2007), puisque les populations qui les utilisent souffrent également de maladies hydriques (diarrhée, gastroentérite).

Les populations de la commune de Lokossa ont leur façon traditionnelle de traiter l'eau avant la consommation. Les méthodes de traitement sont la décantation, la désinfection par l'hypochlorite (eau de Javel) et l'utilisation du sulfate d'alumine (alun). La figure 7 présente les techniques de traitement de l'eau dans le secteur d'étude. 


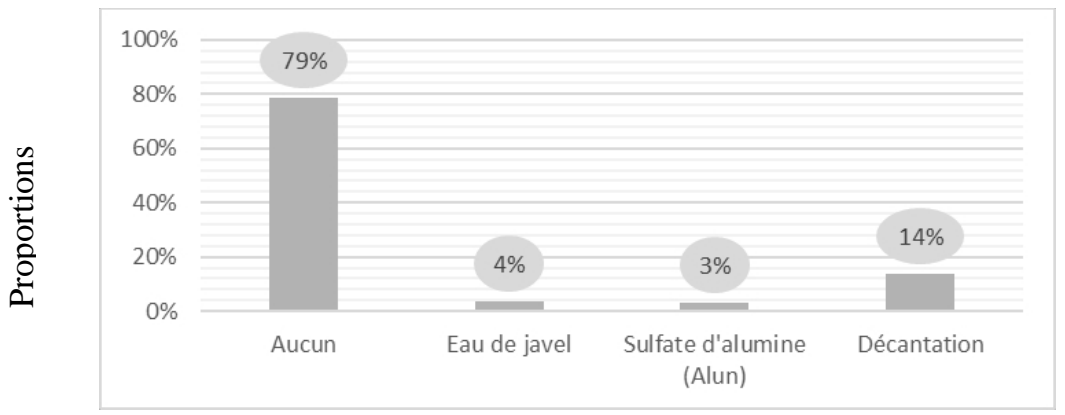

Traitement endogène de l'eau de consommation

Figure 7 : Techniques de traitement de l'eau de consommation dans la commune de Lokossa

Source : Résultats des travaux d'enquête de terrain, (Ahouansè, 2015, 2016, 2017, 2018) et traitements statistiques

L'analyse de la figure 7 montre que $79 \%$ des ménages enquêtés n'utilisent aucun procédé de traitement de l'eau de consommation. Le mode de traitement le plus utilisé dans toute la commune est la décantation (repos prolongé de l'eau sans ajout de quelconque produit) pour faciliter le dépôt des particules non solubles. Mais cela se développe très lentement encore (environ $14 \%$ des enquêtés) dans toute la commune. A cela s'ajoutent ceux qui utilisent l'hypochlorite (eau de Javel) qui font $4 \%$ et ceux qui préfèrent sulfate d'alumine (alun) qui représentent juste $3 \%$.

Il ressort de ces observations que d'une manière générale, les enquêtés ne jugent pas nécessaire de traiter l'eau avant la consommation. La nécessité de potabilisation de l'eau surtout tirée des sources non recommandées pose ainsi le problème de la qualité de l'eau utilisée par la population de la commune de Lokossa pour la boisson comme pour les autres usages domestiques. Sèbo (2014), Babadjidé (2011), Gomez (2010) et Totin (2010) confirment cette préoccupation de la qualité de l'eau en observant que l'une des causes de la morbidité humaine respectivement dans le bassin sudbéninois du fleuve Mono, dans le milieu Itcha-Ifê et dans le bassin sédimentaire côtier du Bénin est l'eau de boisson contaminée par la pratique de la défécation sauvage, de la mauvaise gestion des ordures ménagères et autres déchets et par la pollution chimique due à l'utilisation des engrais et des pesticides. 


\section{3-2 Qualité physico-chimique et bactériologique de l'eau de consommation}

\section{3-2-1 Qualité physico-chimique des eaux prélevées}

Les paramètres mesurés concernent : la température, le potentiel hydrogène, la conductivité et la couleur des échantillons d'eau prélevés à la source. Le tableau II présente ces paramètres.

Tableau II : Caractéristiques physiques des échantillons d'eau prélevés

\begin{tabular}{|c|c|c|c|c|c|c|c|}
\hline $\begin{array}{l}\text { Paramètres } \\
\text { physiques }\end{array}$ & Normes & Observations & $\mathbf{n}$ & Minimum & $\begin{array}{l}\text { Maxi } \\
\text { mum }\end{array}$ & $\begin{array}{l}\text { Moyenn } \\
\text { e }\end{array}$ & $(+/-\mathbf{E T})$ \\
\hline $\begin{array}{l}\text { Températur } \\
\text { e }\left({ }^{\circ} \mathrm{C}\right)\end{array}$ & 25 & $\begin{array}{l}\leq 25 \\
>25\end{array}$ & $\begin{array}{l}0 \\
25\end{array}$ & 29,1 & 29,4 & 29,29 & 0,1 \\
\hline $\begin{array}{l}\mathrm{pH} \quad \text { (Unité } \\
\mathrm{pH})\end{array}$ & $6,5-8,5$ & $\begin{array}{l}<6,5 \\
6,5-8,5 \\
>8,5\end{array}$ & $\begin{array}{l}24 \\
1 \\
0\end{array}$ & 4,02 & 7,3 & 4,97 & 0,99 \\
\hline $\begin{array}{l}\text { Conductivit } \\
\text { é }(\mu \mathrm{S} / \mathrm{cm})\end{array}$ & 2000 & $\begin{array}{l}\leq 2000 \\
>2000\end{array}$ & $\begin{array}{l}25 \\
0\end{array}$ & 46,3 & 1856 & 388,48 & 502,89 \\
\hline $\begin{array}{l}\text { Couleur } \\
\text { (uc) }\end{array}$ & $<15$ & $\begin{array}{l}<15 \\
>15\end{array}$ & $\begin{array}{l}16 \\
9\end{array}$ & 0 & 239 & 27,15 & 66,72 \\
\hline
\end{tabular}

Source : Résultats d'analyse de laboratoire (DGEau, 2018) et traitements statistiques

De l'analyse du tableau II :

- la température tourne autour de $29^{\circ} \mathrm{C}$. Ces valeurs de la température sont au-delà de la valeur de référence $\left(25^{\circ} \mathrm{C}\right)$ fixée par l'OMS pour une eau destinée à la consommation humaine. Une eau dont la température est au-delà de $25^{\circ} \mathrm{C}$, constitue un milieu favorable au développement des microorganismes et donc à la pollution des eaux. Les températures élevées que nous avons retrouvées dans notre étude se rapprochent de celles de 26 à $28^{\circ} \mathrm{C}$ retrouvées à Kandi par Djafarou (2004) et de celles variant entre 25 et $30^{\circ} \mathrm{C}$ retrouvées à Grand-Popo par Makoutodé et al. (1999). Ces résultats sont aussi de même ordre que celles trouvées par Degbey et al. (2008) dans la Commune d'AbomeyCalavi au Bénin où les températures ont varié entre 28,3 et $29,9^{\circ} \mathrm{C}$ avec une moyenne de $28,56^{\circ} \mathrm{C}$.

- le pH des eaux consommées varie de 4,02 à 7,3, avec une moyenne de 4,98 et un écart-type de 0,99 .

- la conductivité varie entre 46,3 et $1856 \mu \mathrm{S} / \mathrm{cm}$, avec une moyenne de $388,87 \mu \mathrm{S} / \mathrm{cm}$ et un écart moyen de 458,11 .

- la couleur de l'eau montre une variation d'un site à un autre allant de 0 et 239 uc. 
L'analyse chimique a consisté à mesurer les ions majeurs et mineurs que sont l'ammonium $\left(\mathrm{NH}_{4}^{+}\right)$, le Nitrate $\left(\mathrm{NO}^{-}\right)$, le Nitrite $\left(\mathrm{NO}^{-}\right)$, le Sulfate $\left(\mathrm{SO}_{2}{ }^{-}\right)$, le Fluore, l'Iodure ( $\left.\mathrm{I}^{-}\right)$, le Fer total, le Chlorure $\left(\mathrm{Cl}^{-}\right)$, les Bicarbonates $\left(\mathrm{HCO}^{-}\right.$ ), le Magnésium $\left(\mathrm{Mg}^{+}\right)$. Le tableau III présente ces paramètres.

Tableau III : Caractéristiques chimiques des échantillons d'eau prélevé

\begin{tabular}{|c|c|c|c|c|c|c|c|}
\hline $\begin{array}{l}\text { Paramètres } \\
\text { chimiques }\end{array}$ & Normes & Observations & $\mathbf{n}$ & Minimum & Maximum & Moyenne & $(+/-\mathbf{E T})$ \\
\hline $\begin{array}{l}\text { Ammonium } \\
\mathrm{NH}_{4}^{+}(\mathrm{mg} / \mathrm{L})\end{array}$ & 0.5 & $\begin{array}{l}\leq 0,5 \\
>0,5\end{array}$ & $\begin{array}{l}24 \\
1\end{array}$ & 0 & 2,58 & 0,36 & 0,69 \\
\hline $\begin{array}{ll}\text { Nitrates } & \mathrm{NO}_{3}{ }^{-} \\
(\mathrm{mg} / \mathrm{L}) & \end{array}$ & 50 & $\begin{array}{l}\leq 50 \\
>50\end{array}$ & $\begin{array}{l}24 \\
1\end{array}$ & 0 & 167,2 & 23,42 & 45,35 \\
\hline $\begin{array}{l}\text { Nitrites } \\
\mathrm{NO}_{2}^{-}(\mathrm{mg} / \mathrm{L})\end{array}$ & 3.2 & $\begin{array}{l}\leq 3,2 \\
>3,2\end{array}$ & $\begin{array}{l}25 \\
0\end{array}$ & 0 & 1,018 & 0,112 & 0,2835 \\
\hline $\begin{array}{l}\text { Sulfates } \\
\mathrm{SO}_{4}{ }^{2-}(\mathrm{mg} / \mathrm{L})\end{array}$ & 500 & $\begin{array}{l}\leq 500 \\
>500\end{array}$ & $\begin{array}{l}25 \\
0\end{array}$ & 0 & 45 & 8,54 & 11,77 \\
\hline Fluorures & 1.5 & $\begin{array}{l}\leq 1,5 \\
>1,5\end{array}$ & $\begin{array}{l}25 \\
0\end{array}$ & 0 & 1,11 & 0,33 & 0,31 \\
\hline Iodure $\mathrm{I}^{-}(\mathrm{mg} / \mathrm{L})$ & - & - & - & 0,02 & 0,73 & 0,078 & 0,05 \\
\hline $\begin{array}{l}\text { Fer total } \\
\mathrm{Fe}^{2+} / \mathrm{Fe}^{3+}(\mathrm{mg} / \mathrm{L})\end{array}$ & 0.3 & $\begin{array}{l}\leq 0,3 \\
>0,3\end{array}$ & $\begin{array}{l}24 \\
1\end{array}$ & 0,04 & 0,4 & 0,11 & 0,099 \\
\hline $\begin{array}{l}\text { Chlorures } \\
(\mathrm{mg} / \mathrm{L})\end{array}$ & 250 & $\begin{array}{l}\leq 250 \\
>250\end{array}$ & $\begin{array}{l}24 \\
1\end{array}$ & 7,1 & 710 & 95,31 & 187,76 \\
\hline $\begin{array}{l}\text { Bicarbonates } \\
\mathrm{HCO}_{3}^{-}(\mathrm{mg} / \mathrm{L})\end{array}$ & - & - & - & 6,1 & 408,7 & 77,66 & 124,033 \\
\hline $\begin{array}{l}\text { Magnésium } \\
\mathrm{Mg}^{2+}(\mathrm{mg} / \mathrm{L})\end{array}$ & 50 & $\begin{array}{l}\leq 50 \\
>50\end{array}$ & $\begin{array}{l}25 \\
0\end{array}$ & 0,973 & 20,41 & 5,39 & 5,28 \\
\hline $\begin{array}{l}\text { Calcium } \\
\mathrm{Ca}^{2+}(\mathrm{mg} / \mathrm{L})\end{array}$ & 100 & $\begin{array}{l}\leq 100 \\
>100\end{array}$ & $\begin{array}{l}25 \\
0\end{array}$ & 3,2 & 78,56 & 25,14 & 26,44 \\
\hline $\begin{array}{l}\text { Dureté } \quad \text { totale } \\
(\mathrm{mg} / \mathrm{L})\end{array}$ & 500 & $\begin{array}{l}\leq 500 \\
>500\end{array}$ & $\begin{array}{l}25 \\
0\end{array}$ & 19,96 & 195,77 & 53,95 & 47,32 \\
\hline Alcalinité (mg/L) & - & & & 9 & 35 & 14,54 & 9,5 \\
\hline
\end{tabular}

Source : Résultats d'analyse de laboratoire (DGEau, 2018) et traitements statistiques 
Les paramètres chimiques à hauts risques pour la santé des populations sont les nitrates et les fluorures.

Au-delà d'un certain seuil de concentration, les nitrates peuvent engendrer, chez les enfants et surtout chez les nourrissons très sensibles à une absorption trop importante, un empoisonnement du sang appelé une méthémoglobinémie ou maladie bleue. Les nitrates ne sont pas nocifs en soi pour la santé. Mais sous l'action d'une bactérie présente dans le corps humain, ils se transforment en nitrites qui eux oxydent l'hémoglobine du sang qui ne peut plus fixer l'oxygène et perturbe la respiration cellulaire.

Le fluor est un élément principal de la prévention des caries dentaires. Cependant une consommation régulière d'eau dont la concentration en ion $\mathrm{F}^{-}$est supérieure à $2 \mathrm{mg} / \mathrm{L}$ peut entraîner des problèmes de fluorose dentaire et même de perte de dents (Ginglinger, 1996). Des concentrations plus élevées peuvent être à l'origine de la fluorose osseuse, beaucoup plus grave, qui se manifeste par la déformation des os surtout chez les enfants.

Les eaux destinées à la consommation humaine doivent respecter des valeurs limites pour être qualifiées de potables et sans danger.

La plupart des eaux consommées dans le secteur d'étude respecte la norme en nitrate sauf un échantillon dont la concentration est de $167,2 \mathrm{mg} / \mathrm{L}$ (puits de Houin-Dessa). Selon l'OMS, la norme maximale à partir de laquelle la dose du nitrate dans l'eau de consommation nuit à la santé humaine est de $50 \mathrm{mg} / 1$ (WHO, 2008). Selon Hirondel (1993), les nitrates, sous l'action des bactéries présentent dans le corps humain, se transforment en nitrites. Ceux-ci oxydent hémoglobine du sang qui ne peut plus fixer l'oxygène ; ce qui perturbe la respiration cellulaire. Même à faible concentration, ils peuvent également engendrer à long terme des cancers chez les adultes lorsqu'ils sont associés à certains pesticides avec lesquels ils forment des composés cancérigènes (Goudot, 2003 ; OMS, 1998).

Quant aux fluorures, des teneurs supérieures à la norme $(1,5 \mathrm{mg} / \mathrm{L})$ font courir le risque accru de fluorose dentaire, selon les directives de l'OMS. Un échantillon (puits de Houin-Tokpa) a une concentration qui est à la limite $(1,11 \mathrm{mg} / \mathrm{L})$.

\section{3-2-2 Qualité bactériologique des eaux consommées}

Les figures 8 et 9 présentent respectivement la qualité bactériologique de l'eau d'une borne fontaine et d'un puits en vue de faire une comparaison de la contamination depuis la source au stockage en passant par le transport. La figure 8 donne la qualité bactériologique de l'eau de la borne fontaine de Aligoudo (Arrondissement de Agamè), prélévée à la source, pendant le transport et au stockage. 


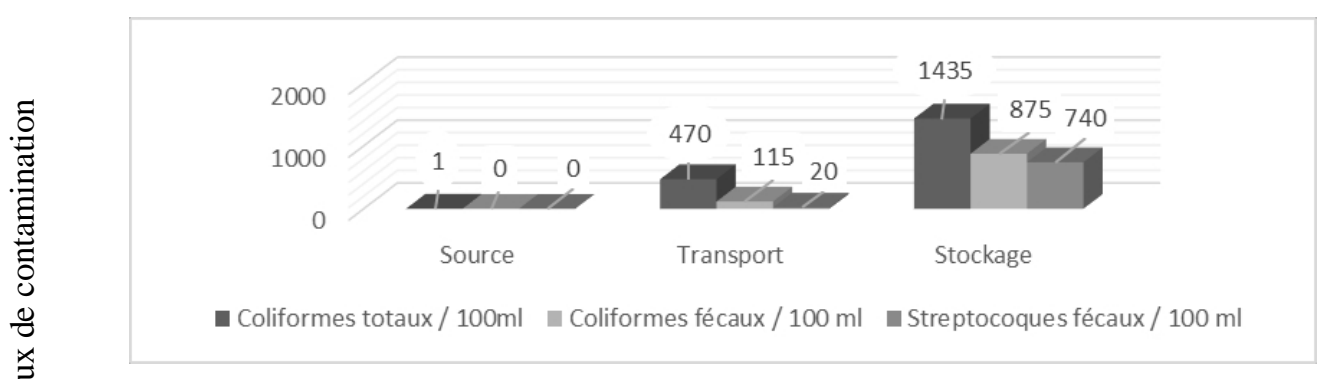

Qualité de l'eau à la source, au cours du transport et pendant le stockage

Figure 8 : Qualité bactériologique de l'eau de BF de Aligoudo (Arrondissement de Agamè), à la source, au cours du transport et pendant le stockage

Source : Résultats d'analyse de laboratoire (DGEau, 2018) et traitements statistiques

De l'observation du graphe 8 , on remarque une contamination progressive en coliformes totaux, coliformes fécaux et streptocoques fécaux de l'eau du BF de Aligoudo entre le transport et le stockage. Les valeurs de la contamination sont très élevées au stockage : 1435/100 $\mathrm{ml}$ pour les coliformes totaux, $875 / 100 \mathrm{ml}$ pour les coliformes fécaux et $740 / 100 \mathrm{ml}$ pour streptocoques fécaux. L'eau de la borne fontaine est potable à la source parce que ne présentant aucune contamination.

La figure 9 met en exergue la qualité bactériologique de l'eau du puits de Ablodé-Ahouassa (Arrondissement de Koudo), prélévée à la source, pendant le transport et au stockage.

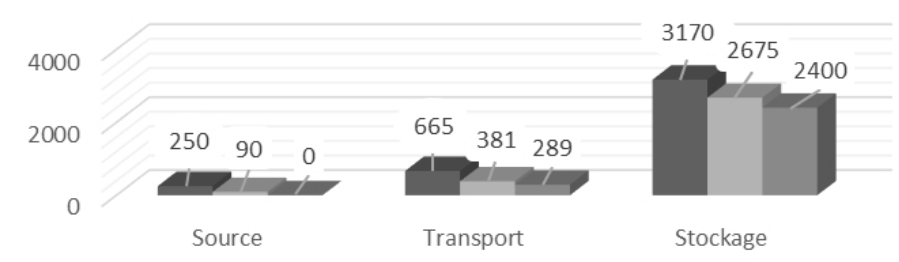

- Coliformes totaux / 100ml a Coliformes fécaux / $100 \mathrm{ml} \mathrm{a} \mathrm{Streptocoques} \mathrm{fécaux} \mathrm{/} 100 \mathrm{ml}$

Qualité de l'eau à la source, au cours du transport et pendant le stockage

Figure 9 : Qualité bactériologique de l'eau du puits de Ablodé-Ahouassa (Arrondissement de Koudo), à la source, au cours du transport et pendant le stockage

Source : Résultats d'analyse de laboratoire (DGEau, 2018) et traitements statistiques

De la lecture de la figure 9, il ressort une contamination croissante de l'eau du puits de Ablodé-Ahouassa de la source au stockage où on a des valeurs maximales (3170/100 ml pour les coliformes totaux, 2675/100 ml pour les coliformes fécaux et $2400 / 100 \mathrm{ml}$ pour streptocoques fécaux). La consommation d'une telle eau comporterait de graves risques à l'organisme humain. Il faut noter une absence de streptocoques fécaux à la source ; ce qui traduit une contamination récente de l'eau à la source. Les fortes 
concentrations enregistrées lors du transport et à domicile au moment du stockage de l'eau s'explique tout simplement par les diverses manipulations faites dans l'eau. Le récipient de prise de l'eau, les mains chargées de bactéries lors de la prise de l'eau et l'hygiène peu assurée des récipients de conserve de l'eau sont autant d'éléments qui peuvent altérer la qualité de l'eau de consommation surtout à domicile. Ce résultat est confirmé par Cairncross (2003) qui situe la pollution de l'eau à trois niveaux fondamentaux (source, transport, stockage).

\section{3-3 Effets sanitaires de l'eau de consommation}

L'eau constitue l'un des paramètres environnementaux les plus importants de la morbidité humaine à travers les maladies hydriques. Les maladies hydriques sont celles causées par l'eau contaminée par des déchets humains, animaux ou chimiques. Les maladies hydriques comprennent entre autres le choléra, la diarrhée, le paludisme, la fièvre typhoïde, l'hépatite A et E, etc. (OMS, 2007). D'après les statistiques de cette organisation, chaque jour, en moyenne 6000 personnes meurent dans le monde à cause de maladies diarrhéiques. Les enfants, les femmes enceintes et les personnes âgées sont les âmes les plus exposées aux effets de ces maladies dans les pays les moins développés. La raison principale de cette situation catastrophique est la pauvreté. L'éducation à l'hygiène et le développement de systèmes de traitement des déchets et d'assainissement des eaux permettent de circonscrire à long terme les épidémies déclenchées par ces maladies (Lavallée, 2001).

La présente thèse s'intéresse aux maladies hydriques: les maladies diarrhéiques (diarrhées fébriles et diarrhées avec déshydratation) et le paludisme qui sont des maladies liées à l'eau et provoquées par la consommation de l'eau souillée ou la cohabitation avec des points d'eau mal entretenus. En effet, la pollution de l'eau est responsable de nombreux cas de diarrhées de même que son abondance dans certaines conditions et sous certaines formes de gestion est cause de paludisme.

\section{3-3-1 Prévalence des maladies diarrhéiques dans la commune de Lokossa}

La figure 10 indique la prévalence des maladies diarrhéiques par Arrondissement dans le secteur d'étude. 


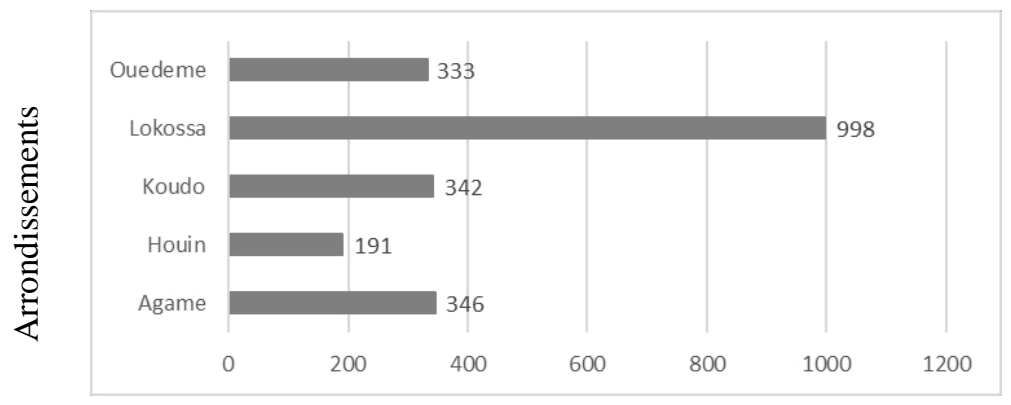

Prévalence des maladies diarrhéiques

Figure 10 : Moyennes annuelles de la prévalence des maladies diarrhéiques par Arrondissement dans la Commune de Lokossa de 2002 à 2018

Source : SNIGS, 2002 à 2018 et traitements statistiques

L'observation des cas de maladies diarrhéiques au niveau du Système National Intégré de Gestion Sanitaire (SNIGS) fait apparaître une distribution spatiale inégale des affections. En effet, en considérant l'ensemble des arrondissements de la Commune, la prévalence des maladies diarrhéiques est en moyenne de 442 par année. Quant aux Arrondissements individuellement pris, l'Arrondissement de Lokossa est la plus affectée (998 cas) tandis que l'Arrondissement de Houin est la moins affectée (191 cas). Le taux de desserte seul ne saurait être mis en cause comme au niveau de la dépression des Tchi (Azonhè, 2009). Cependant, les difficultés d'accès à l'eau pourraient aussi justifier la prévalence élevée dans certaines localités. Ces raisons justifient également les disparités observées au niveau des nombres d'épisodes diarrhéiques dans les ménages. Balthazar, et al., (1988) confirment que «les agents infectieux de la diarrhée sont propagés par la voie féco-orale, notamment par ingestion d'eau ou d'aliments contaminés par les selles ou par contact direct avec des selles infectées ».

\section{3-3-2 Prévalence du paludisme dans la commune de Lokossa}

La figure 11 indique la prévalence du paludisme par Arrondissement dans le secteur d'étude.

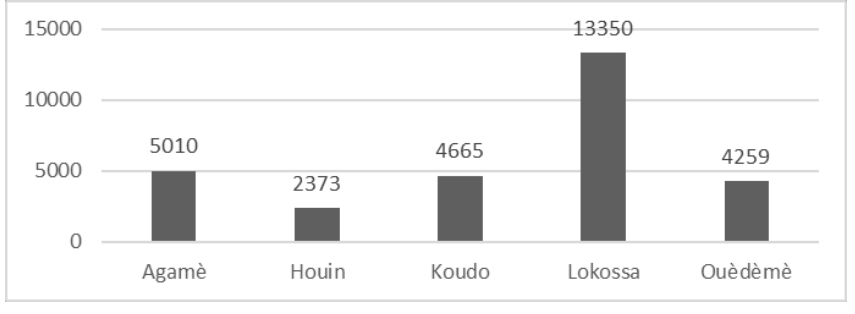

Arrondissements

Figure 11 : Moyennes annuelles de la prevalence du paludısme par Arrondissement dans la Commune de Lokossa de 2002 à 2018

Source : SNIGS, 2002 à 2018 et traitements statistiques 
L'observation des cas de paludisme au niveau du Système National Intégré de Gestion Sanitaire (SNIGS) fait apparaître une distribution spatiale inégale des affections. En effet, en considérant l'ensemble des arrondissements de la Commune, la prévalence des maladies diarrhéiques est en moyenne de 5930 cas par année. Quant aux Arrondissements individuellement pris, l'Arrondissement de Lokossa est la plus affectée (13350 cas) tandis que l'Arrondissement de Houin est la moins affectée (2373 cas). $\mathrm{La}$ présence permanente d'eau aux alentours de certaines sources d'approvisionnement en eau de consommation constitue des gîtes larvaires importants qui expliquent la prévalence du paludisme dans la Commune. En effet, des puits peu profonds et sans margelles ont été observés dans la zone d'étude. Certains de ces puits ont connu une détérioration, mais les margelles de la plupart se sont enfoncées dans le sol en raison de la nature de ce dernier. D'autres puits ont alors été recouverts d'une dalle en béton pour empêcher l'eau de ruissellement d'y pénétrer. Mais les pratiques de puisage participent à la permanence de l'eau aux alentours de l'ouvrage. Mouchet et al. (1988) montre que «Anopheles gambiae est très abondants dans toutes les collections d'eau de surface, alimentées par les forages ».

\section{3-3-3 Temporalité des maladies hydriques}

La prévalence des maladies hydriques évolue dans le temps au rythme des périodes climatiques et touche différemment les différentes localités du secteur d'étude. La figure 12 montre l'évolution saisonnière des deux principales maladies hydriques recensées dans la commune de Lokossa.

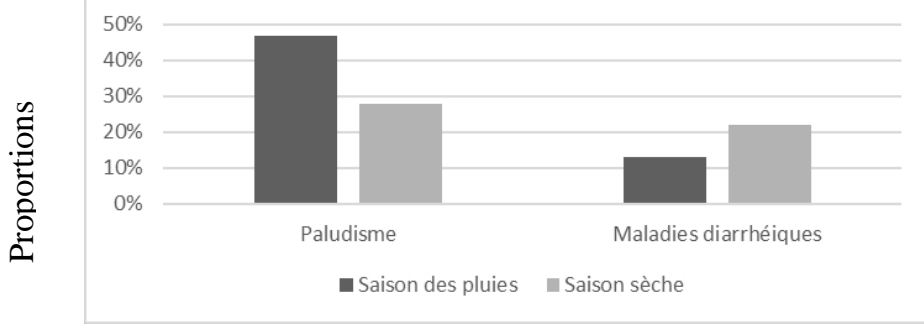

Maladies hydriques selon les saisons

Figure 12 : Etat sanitaire des ménages selon les Arrondissements de la Commune Source : Résultats des travaux d'enquête de terrain, (Ahouansè, 2018) et traitements statistiques

L'observation de la figure 12 montre qu'en saison sèche, la prévalence des maladies diarrhéiques est élevée et diminue en saison des pluies. C'est le contraire qui s'observe au niveau du paludisme.

$\mathrm{Au}$ cours de la saison sèche, les populations qui n'ont pas un accès facile aux sources d'eau recommandées font recours à celles non recommandées (puits traditionnels et eaux de surfaces) dont la baisse de 
niveau accentue la concentration des agents pathogènes. Les eaux des rivières deviennent les principales sources d'approvisionnement des populations et sont surtout consommées par les enfants comme eaux de boisson. Ce qui peut justifier le taux élevé des maladies diarrhéiques. A ceci s'ajoutent les problèmes d'hygiène et d'assainissement et la mauvaise manipulation de l'eau de consommation qui pourraient expliquer les épisodes de maladies diarrhéiques en toute saison. Ces résultats corroborent ceux de Sèbo (2014), dans le bassin géographique sud-béninois du fleuve Mono, Agbossou (2001) constate que les populations de Lokossa ont été beaucoup éprouvées par les gastro-entérites de janvier à avril 2000, période correspondant à la saison sèche. Des observations similaires sont faites par Orékan (2000) à Gomé et à Tré, par Azonhè (2009) dans la dépression des Tchi et par Babadjidé (2010) dans le bassin du Mono.

Quant au paludisme, pendant la saison des pluies, les réservoirs (citernes à ciel ouvert, jarres mal entretenus) utilisés pour la collecte des eaux de pluie, les eaux stagnantes un peu partout, les dépotoirs sauvages, facilitent la multiplication des moustiques (anophèles) qui transmettent à l'organisme humain le plasmodium falciparum, germe responsable du paludisme. La mauvaise gestion des eaux de pluies contribue au développement des larves de moustiques. A cela s'ajoutent les problèmes d'assainissement autour des points d'eau qui favorisent la multiplication des moustiques responsables du paludisme. C'est le cas des forages artésiens qui par leur aménagement précaire forment des mares. En effet, la plupart des vecteurs du paludisme évoluent dans des milieux marqués par la présence de l'eau.

\section{Conclusion}

Les résultats permettent de dire que les eaux que consomment les populations de la commune de Lokossa sont polluées à la source, mais surtout entre le transport et le stockage. L'étude a montré une forte prévalence des maladies hydriques dans la commune de Lokossa. Ces affections sont surtout les maladies diarrhéiques et le paludisme. Les statistiques épidémiologiques de la Zone Sanitaire de Lokossa montrent que les maladies liées à l'eau telles que le paludisme et les diarrhées occupent toute l'année un pourcentage important des principales affections reçues en consultation dans les formations sanitaires. Ces maladies d'origine hydrique sont dans le cadre de cette étude des maladies provoquées par la consommation d'eau contaminée par des fèces animales ou humaines, qui contiennent des microorganismes pathogènes ou par la cohabitation avec des points d'eau mal entretenus (citernes à ciel ouvert, forage artésien formant des mares favorables au développement des larves de moustique). L'amélioration de la santé de la population de la commune de Lokossa suppose, outre l'amélioration de la qualité de l'eau à usage domestique, la modification de certains comportements. Ce changement passe 
par une étude des comportements existants, une identification des freins à leur modification et par la définition d'objectifs de changement. Un partenariat entre les différents acteurs concernés, l'utilisation de moyens d'animation sanitaire adéquats et des méthodes participatives concourent à l'adoption de ces nouveaux comportements. Toutefois, une plus grande décentralisation et responsabilisation des acteurs locaux ne doit pas enlever à l'Etat sa responsabilité générale, notamment dans la mission qui lui revient d'assurer à tous le droit à l'eau potable.

Pour finir, l'insuffisance quantitative et qualitative de l'eau associée aux comportements peu hygiéniques des populations engendre des maladies hydriques dont les diarrhées et le paludisme, lesquelles ont des impacts considérables sur la production et par conséquent sur les revenus des populations. La résolution de ces problèmes nécessite une bonne communication pour le changement de comportement de la communauté et un renforcement en infrastructure d'approvisionnement en eau potable.

\section{References:}

1. ABéNor, (2003) : Eaux minérales naturelles et eaux de source : Normes béninoises. Spécifications et critères de qualité. $1^{\text {ère }}$ édition, Bénin, 17 p.

2. Agbossou, E., (2001) : Impacts sanitaires de l'exploitation agricole des sols inondables sur l'environnement humain des zones humides du sud-Bénin : cas des entités territoriales de Lokossa et d'Athiémé au Bénin. 23 p.

3. Agossou, N., (1991) : Disparités et aménagement en Afrique, la modernisation de Lokossa au Bénin. Fragments de géosociographique. Québec : Ecole des gradués de l'université Laval, Thèse de Ph. D. de géographie, $421 \mathrm{p}$.

4. Agossou, N., (2014) : Initiation à la méthode de travail (Licence, Master, Doctorat). EPA/CERADE, 246 p. Coll. Initiation $\mathrm{n}^{\circ} 3,2^{\mathrm{e}}$ édition refondue et augmentée.

5. Agossou, N., (2016) : Fondamentaux de Géographie du Bénin. $2^{\mathrm{e}}$ édition, Editions Populaires Africaines, Porto-Novo, 216 p.

6. Ahouansè, S., D., M., (2006) : Etude de l'aménagement et de la gestion des points d'eau de la commune de Lokossa : Impacts sur la vie socioéconomique des populations. Mémoire de Maîtrise de Géographie, FLASH/UAC, Abomey-Calavi, 113 p.

7. Ahouansè, S., D., M., (2011) : Impacts environnementaux de la gestion de l'eau dans le département du Mono en République du Bénin (Afrique de l'ouest). Mémoire de DEA en Gestion de l'Environnement, EDP/UAC, Abomey-Calavi, 92 p. 
8. Ancelle, T., (2011) : Statistique Epidémiologique, 2ème édition, Coll. Sciences Fondamentales, Maloine, Paris, 9 p.

9. Azonhè, T., H., S., N., (2009) : Analyse des déterminants environnementaux du paludisme et des maladies diarrhéiques chez les populations du secteur agricole dans la dépression des Tchi au sud du Bénin. Thèse de doctorat unique. UAC, Abomey-Calavi, 235 p.

10. Babadjidé, C., L., (2008) : Gestion endogène de l'eau et santé des populations dans la commune de Savè : cas de l'arrondissement de Bessé. Mémoire de DEA, UAC, Abomey-Calavi, 105 p.

11. Babadjidé, C., L., (2011): Etude de l'influence de la pollution hydrique sur la santé humaine dans le bassin du fleuve Mono au Bénin. Thèse de doctorat, EDP/GEN/FLASH/UAC, Abomey-Calavi, 208 p. + annexes.

12. Balthazar, J., C., et Solon, F., S., (1988): Disposal of faeces of children under two years old and diarrhoea. A case control study. In International Journal Epidemiology, Suppl. 2, 18, 4, pp. 16-19.Boukir, 1997.

13. Blalogoe, P., (2002): L'eau et la santé publique en milieu de transition: Etude de cas de la commune de Glazoué. Mémoire de maîtrise en géographie, Abomey-Calavi, $108 \mathrm{p}+$ annexes.

14. CAE, (2010) : Approvisionnement en eau potable au Bénin : problèmes et perspectives. Rapport ; $68 \mathrm{p}$.

15. Cairncross, S., (2003): Measuring the health impact of water and sanitation: $1-4$.

16. Codjia, D., H., F., (2018) : Facteurs environnementaux et prévalence des maladies liées à l'eau dans la commune de Tori-Bossito (République du Bénin, Afrique de l'Ouest), Thèse pour l'obtention du diplôme de Doctorat Unique de l'Université d'Abomey-Calavi, EDP, FASHS, UAC, Abomey-Calavi, 277 p.

17. Dégbey, C., Makoutodé, M., Ouendo, E., M., Fayomi, B., de Brouwer, C., (2008) : La qualité de l'eau de puits dans la Commune d'AbomeyCalavi au Bénin. Environnement Risque Santé 2008 ; 7 : 279-283. Doi : $10.1684 /$ ers.0158.

18. DGEau, (2006): Rapport de synthèse de l'atelier national sur la définition d'une gestion pérenne des AEV. Cotonou, MMEE, 12 p.

19. DGEau, (2008) : Politique Nationale de l'Eau. Version définitive. Rapport de Synthèse, MEE, Cotonou, $51 \mathrm{p}$.

20. DGEau, (2010) : Evaluation du processus de professionnalisation de la gestion des AEV au Bénin. Rapport final, $131 \mathrm{p}$.

21. DG-Eau, (2014) : Accès à l'eau potable : le Benin atteint les OMD http://www.afwa-hq.org/index.php/fr/actualites/item/829-acces-a-1eau-potable-le-benin-atteint-les-omd. 
22. Djafarou, A., (2004) : La contribution à l'évaluation des risques liés aux usages domestiques de l'eau dans la commune de Kandi. Mémoire de DESS, IMSP, Cotonou, $69 \mathrm{p}$.

23. Ginglinger, B., (1996) : Le point sur le fluor en France en 1996. Thèse Chir. Dent., Strasbourg, p 39.

24. Gomez, C., A., (2010) : Qualité des eaux de consommation et l'état de santé des populations en milieu Itcha-Ifè dans le département des Collines au Bénin. Thèse de Doctorat Unique de géographie, EDP/FLASH/UAC, Abomey-Calavi, 265 p.

25. Goudot, S., (2003) : Les nitrates et pollution. Disponible sur : http://fr.wikipedia.org/wiki/Nitrate (consulté le 18 février 2010).

26. Hedible, C., S., (2007) : Perceptions et stratégies d'adaptation des communautés rurales du département de l'Atlantique face à la dégradation de la qualité de l'eau de consommation. Thèse de Doctorat Unique de géographie, UAC, Abomey-Calavi, 215 p.

27. Hirondel, J., (1993) : Les méthémoglobinémies du nourrisson. Données nouvelles. Cahier de Nutrition et de Diététique, 28, 341-9.

28. Houssou, S., C., Vissin, E., W., et Perard, J., (2006) : Variabilité climatique et pathologie dans le département du mono (Bénin, Afrique de l'Ouest). Colloque AIC, Epernay, 8 p.

29. INSAE, (2016) : Effectifs de la population des villages et quartiers de ville du Bénin (RGPH-4, 2013). Cotonou, 85 p.

30. Kotcharé, K., P., Ahouansè, S., D., M., Dako Kpatcha, S., et Houssou, S., C., (2017) : Les pratiques en eau, hygiène et assainissement en milieu scolaire : cas des écoles primaires publiques de la commune d'Abomey-Calavi, $\mathrm{VI}^{\mathrm{e}}$ colloque des sciences, cultures et technologies, Conseil scientifique, UAC, Abomey-Calavi, 726 p.

31. Lalèyè, E., S., (2016) : Identification et analyse des déterminants de la contamination des eaux des sources et forages artésiens dans les communes de Zagnanado et de Zogbodomey au Bénin. Thèse pour l'obtention du diplôme de Doctorat Unique de l'Université d'AbomeyCalavi, EDP, FASHS, UAC, Abomey-Calavi, 269 p.

32. Lavallee, M., (2001) : Les déterminants du choléra. Développement et Santé, $\mathrm{n}^{\circ} 152$.

33. Makoutodé, M., Assani, A. K., Ouendo, E-M., Agueh, V. D., Diallo, P., (1999) : Qualité et mode de gestion de l'eau de puits en milieu rural au Bénin: cas de la sous-Préfecture de Grand-Popo. Médecine d'Afrique Noire. 46 (11) : 528-534.

34. Marien, B., et Beaud, J-P., (2003) : Guide pratique pour l'utilisation de la statistique en recherche : le cas des petits échantillons. Réseau sociolinguistique et dynamique des langues, Agence universitaire de la Francophonie, Québec, 44 p. 
35. Mialo, E. S., (2016) : Eau de boisson et maladies hydriques dans la commune de Lalo au Sud-Bénin. Thèse pour l'obtention du diplôme de Doctorat Unique de l'Université d'Abomey-Calavi, EDP, FASHS, UAC, Abomey-Calavi, $270 \mathrm{p}$.

36. Mouchet, J., Carnevale, P., (1988) : Le paludisme, composante de l'environnement africain. ORSTOM, Actualités ; 1988 ; 20, I-VIII p.

37. Odoulami, L., (2009) : La problématique de l'eau potable et la santé humaine dans la ville de Cotonou. Thèse de Doctorat Unique, EDP/FLASH/CUSTE, UAC, Abomey-Calavi, $230 \mathrm{p}$.

38. OMS, (1998): La santé pour tous ? In le courrier $\mathrm{N}^{\circ} 108$, revue bimestrielle, Genève, publication Offset, pp 60-87, 100 p.

39. OMS, (1998): Nitrate and nitrite, guideline for drinking-water quality. Vol. 1 : Recommandations, WHO pp 8-10.

40. OMS, (2002) : Rapport sur la santé dans le monde 2002. Genève, 180

41. OMS, (2007): Weekly epidemiological report - Relevé épidémiologique hebdomadaire, $82: 273-84$.

42. Orékan, V., (2000) : Impacts de l'exploitation des bas-fonds sur la santé des populations : cas des sous-préfectures de Dassa-Zoumè et de Glazoué dans le département des Collines au Bénin. Mémoire de DEA, EDP/GENV/FLASH/UAC, Abomey-Calavi, 79 p.

43. Sèbo, E., (2014) : Analyse des facteurs de différenciation de la morbidité diarrhéique dans le bassin géographique sud-Béninois du fleuve mono (Afrique de l'ouest). Thèse de doctorat, EDP/GENV/FLASH/UAC, Abomey-Calavi, 339 p.

44. Sohounou, M., (2018) : Qualité de l'eau de boisson et risques toxicologiques dans la commune d'Allada au Bénin. Thèse pour l'obtention du diplôme de Doctorat Unique de l'Université d'AbomeyCalavi, EDP, FASHS, UAC, Abomey-Calavi, 211 p.

45. Totin, V. S. H. (2010) : Sensibilité des eaux souterraines du bassin versant sédimentaire côtier du Bénin à l'évolution du climat et aux modes d'exploitation: Stratégies de gestion durable. Thèse de Doctorat Unique, EDP/UAC, Abomey-Calavi. 272 p.

46. WHO (2008) : Guidelines for drinking-water quality : Incorporating 1 st and 2nd addenda. Vol.1, Recommendations. - 3rd éd., Geneva, $668 \mathrm{p}$ 